\title{
Clinical and hemodynamic influences of prophylactic tricuspid annuloplasty in mechanical mitral valve replacement
}

\author{
Heemoon Lee, MD, ${ }^{a}$ Kiick Sung, MD, PhD, ${ }^{a}$ Wook Sung Kim, MD, PhD, ${ }^{\text {a }}$ Young Tak Lee, MD, PhD, \\ Sung-Ji Park, MD, PhD, ${ }^{\mathrm{b}}$ Keumhee Chough Carriere, $\mathrm{PhD},{ }^{\mathrm{c}, \mathrm{d}}$ and Pyo Won Park, MD, PhD ${ }^{\mathrm{a}}$
}

\begin{abstract}
Objective: The aim of this study was to evaluate the long-term clinical and hemodynamic influences of prophylactic tricuspid annuloplasty (TAP) in patients with less-than-moderate tricuspid regurgitation (TR) who underwent mitral valve replacement (MVR).

Methods: Between November 1994 and December 2010, 293 patients with less-than-moderate TR who underwent primary mechanical MVR were categorized into 2 groups: TAP $(\mathrm{n}=151)$ or no TAP $(\mathrm{n}=142)$. The median age was 51 years (quartile (Q)1-Q3, 43-59 years). The cause of valve pathology was rheumatic in $92.5 \%$ of patients $(n=271)$. The prevalence of preoperative atrial fibrillation was in $73.0 \%$. Using propensity score matching based on demographic information, 91 TAP patients could be matched to 91 no TAP patients. Median follow-up duration was 107 months (Q1-Q3, 76-162 months).
\end{abstract}

Results: There was no early mortality in either group. Early morbidities, including heart block were not different between groups. Although overall survival and freedom from cardiac-related mortality did not differ between groups ( $P=.519$ and $P=.115$, respectively), freedom from recurrence of moderate or higher TR grade were significantly higher in the TAP group $(P=.043)$. In subgroup analyses, these group differences of TAP were especially prominent in patients with sinus rhythm compared with patients with atrial fibrillation at discharge $(P=.047$ vs $P=.460)$.

Conclusions: Prophylactic TAP for patients with less-than-moderate TR grade who underwent mechanical MVR can prevent late TR progression without increasing early surgical risks. Longer-term follow-up is required to determine the clinical beneficial effect of prophylactic TAP. (J Thorac Cardiovasc Surg 2016;151:788-95)

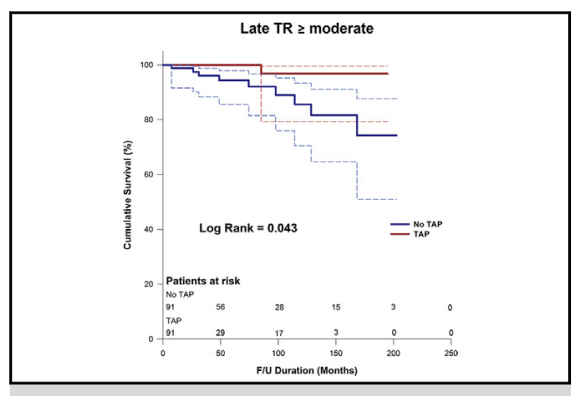

Kaplan-Meier curves for moderate-or-greater late tricuspid regurgitation (TR) in the matched TAP and no TAP groups.

\section{Central Message}

Prophylactic TAP for patients with less-thanmoderate TR who underwent mechanical MVR can prevent late TR progression.

\section{Perspective}

Our study demonstrated that prophylactic tricuspid annuloplasty (TAP) with mechanical mitral valve replacement prevents late recurrence of tricuspid regurgitation without increasing early surgical risk. Although prophylactic TAP showed a marginal significance in clinical benefits for patients with less-thanmoderate tricuspid regurgitation, the beneficial effect of TAP would be more prominent in longer-term follow-up.

See Editorial Commentary page 796.
Severe tricuspid regurgitation (TR) or even moderate TR after cardiac surgery is associated with a poor prognosis and has an adverse influence on survival. ${ }^{1-3}$ Current guidelines

\footnotetext{
From the ${ }^{\mathrm{a}}$ Department of Thoracic and Cardiovascular Surgery and ${ }^{\mathrm{b}}$ Division of Cardiology, Department of Medicine, and ${ }^{\mathrm{d}}$ Biostatistics and Clinical Epidemiology Center, Samsung Medical Center, Sungkyunkwan University School of Medicine, Seoul, Korea; and ${ }^{\mathrm{c} D e p a r t m e n t}$ of Mathematical and Statistical Sciences, University of Alberta, Edmonton, Alberta, Canada.

Read at the 41st Annual Meeting of The Western Thoracic Surgical Association, Whistler, British Columbia, Canada, June 24-27, 2015.

Received for publication July 16, 2015; revisions received Oct 20, 2015; accepted for publication Oct 26, 2015; available ahead of print Jan 9, 2016.

Address for reprints: Pyo Won Park, MD, PhD, Department of Thoracic and Cardiovascular Surgery, Samsung Medical Center, Sungkyunkwan University School of Medicine, 81 Irwon-ro, Gangnam-gu, Seoul 135-710, Korea (E-mail: pwpark@ skku.edu).

0022-5223/\$36.00

Copyright (c) 2016 by The American Association for Thoracic Surgery

http://dx.doi.org/10.1016/j.jtcvs.2015.10.104
}

recommend tricuspid valve repair for severe TR associated with mitral valve disease requiring mitral valve surgery (class I). Tricuspid valve repair is suggested for TR of lesser severity during mitral valve surgery for patients with tricuspid annular dilatation (class IIa), ${ }^{4}$ prior evidence of right-side heart failure (class IIa), or pulmonary hypertension (class IIb). ${ }^{5}$ Nevertheless, there are still controversies and discrepancies in concomitant tricuspid

Scanning this $\mathrm{QR}$ code will take you to the article title page.

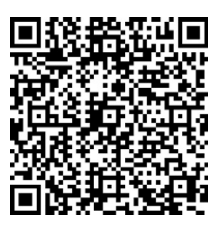




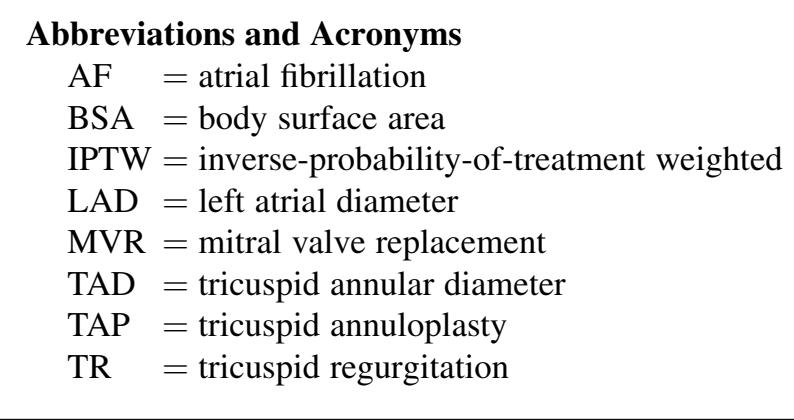

annuloplasty (TAP) with mitral valve surgery. ${ }^{6,7}$ Several authors suggested concomitant TAP with mitral valve surgery can be performed safely and effectively. ${ }^{8,9}$ However, there have been few reports on the results of concomitant TAP with mitral valve surgery in less-thanmoderate TR. ${ }^{8,10,11}$ Further, these reports included a mixed population or only early results or a preliminary result from a subgroup analysis. Furthermore, reports on long-term follow-up data are lacking. The aim of our study was to evaluate early and late clinical outcomes of concomitant TAP in patients who underwent isolated mechanical mitral valve replacement (MVR) with lessthan-moderate TR.

\section{PATIENTS AND METHODS Study Population}

We retrospectively reviewed data from November 1994 to December 2010 for 490 patients who underwent primary MVR with mechanical prosthesis at our hospital. A total of 147 patients with moderate $(+2)$ or greater TR were excluded. Among the remaining 343 patients, 50 patients with endocarditis, concomitant coronary bypass grafting, or history of previous cardiac surgery were excluded. The remaining 293 patients were then classified into 2 groups: TAP $(n=151)$ or no TAP $(n=142)$ (Figure 1$)$. Median age was 51 years (quartile (Q) 1-Q3, 43-59 years). The cause of valve pathology was rheumatic in 271 patients $(92.5 \%)$. The prevalence of preoperative atrial fibrillation $(\mathrm{AF})$ was $73.0 \%(\mathrm{n}=214)$. The study protocol was approved by the hospital's institutional review board, which waived the requirement for patient consent.

\section{Surgical Procedures}

All operations were performed through standard median sternotomy. Cardiopulmonary bypass was performed using bicaval cannulation. Myocardial protection was achieved with intermittent cold blood cardioplegia, usually via an antegrade or retrograde route. Retrograde cardioplegia was infused by direct coronary sinus cannulation in patients with significant aortic regurgitation. Bileaflet mechanical valves were implanted in all patients. The concomitant modified Cox-maze III procedure has been performed in patients with AF since the late 1990s. The technique of maze procedure in our hospital was described in previously published reports. ${ }^{12-14}$

In patients with sinus rhythm and gross right atrial enlargement, the right atrium was opened to observe tricuspid valve annular dilatation. TAP was performed in patients with tricuspid valve annular dilatation

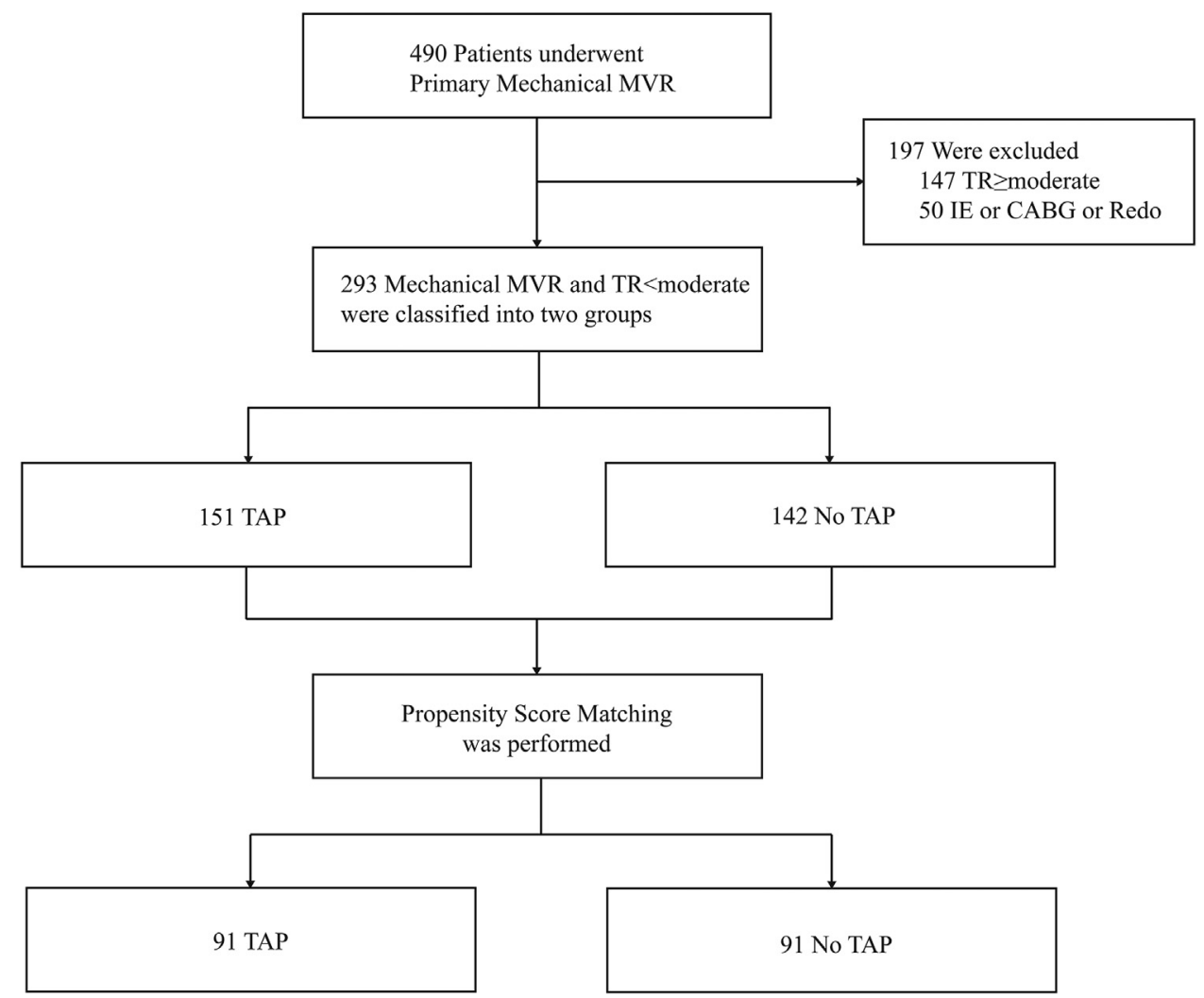

FIGURE 1. Flow diagram of patients enrollment criteria for the study. $M V R$, Mitral valve replacement; $T R$, tricuspid regurgitation; $I E$, infective endocarditis; $C A B G$, coronary artery bypass grafting; $T A P$, tricuspid annuloplasty. 
and mild TR. When AF was associated, we attempted to perform TAP regardless of TR grade and annular dilatation at the time of opening of the right atrium for maze procedure. These surgical strategies for prophylactic TAP may not have been strictly applied to some patients who underwent the operation during the earlier period of our study. Of the propensity score-matched patients in the TAP group, 60 patients $(65.9 \%)$ underwent ring annuloplasty and 31 patients $(34.1 \%)$ underwent suture annuloplasty. For the suture annuloplasty, the modified De Vega method was used for 26 patients $(83.9 \%)$ and the Kay method was used for 5 patients $(16.1 \%)$. For the modified De Vega method, 2-0 monofilament sutures with pledgets at both ends were used from the midportion of septal leaflet to anteroseptal commissure. Two or 3 pledgets were also inserted on the annuloplasty suture to prevent tissue cut-through. Suture annuloplasty was performed mostly during the earlier period of our valve surgery.

\section{Follow-up}

Median follow-up duration was 107 months (Q1-Q3, 76-162 months). In the TAP group follow-up was 91 months (Q1-Q3, 68-122 months) and in the no TAP group follow-up was 151 months (Q1-Q3, $82-188$ months $)(P<.001)$. The primary end point of the study was all-cause mortality. Early mortality was defined as death during hospitalization or within 30 days after the operation. Late mortality was defined as death occurring more than 30 days postoperation. Cause of death was classified as cardiac-related (eg, sudden death, anticoagulation-related neurologic complications, heart failure, or myocardial infarction) or noncardiac. ${ }^{15}$ The secondary end points of the study were the presence of significant moderate-or-greater TR on late follow-up echocardiographic evaluation and tricuspid valve-related events. Tricuspid valve-related events included reoperation for TR, right heart failure, or permanent pacemaker insertion.

Survival follow-up data were available for all patients. Data for $74 \%$ of patients followed-up by our hospital were acquired by reviewing medical records. Data for $5 \%$ of patients followed-up by other hospitals were collected by direct telephone interviews with patients or their families. The remaining $21 \%$ of patients were lost to follow-up, but we confirmed their survival from the National Registry database.

\section{Echocardiographic Evaluation}

Two-dimensional echocardiography with Doppler was performed before discharge and at 1,3,5, and $>7$ years after surgery. Echocardiographic assessments after discharge were obtained in $83.6 \%$ of patients at 1 year, $70.9 \%$ at 3 years, $61.6 \%$ at 5 years, and $57.6 \%$ at 7 years. Left ventricular end-systolic and diastolic dimensions and left atrial diameter (LAD) were measured in the parasternal view based on American Society of Echocardiography guidelines. ${ }^{16}$ Left ventricular ejection fractions were calculated using Simpson's biplane method. The tricuspid annulus was measured in the transthoracic apical 4-chamber view in late diastole at the time of maximal tricuspid opening. ${ }^{17}$ The degree of TR was assessed using the vena contracta width and the ratio of the maximal jet area to the corresponding right atrial area averaged in the parasternal and apical views. TR grade was assessed with Doppler echocardiography according to current standard guidelines. ${ }^{5,18}$ The severity of TR was graded on a scale from 0 to $4(0=$ no or minimal, $1=$ mild, $2=$ moderate, $3=$ moderate to severe, and $4=$ severe) ${ }^{10}$

\section{Statistical Analysis}

Categorical variables are presented as frequencies and percentages, and continuous variables as medians (Q1-Q3). To balance the distribution of baseline risk factors between the groups, propensity score matching was performed. The propensity score was obtained by a multiple logistic regression based on the following preoperative baseline characteristics: age, sex, diabetes mellitus, hypertension, stroke, serum creatinine level, New York Heart Association functional class, and preoperative AF. A total of 91 patients undergoing MVR with TAP were matched on a 1-to-1 manner with patients undergoing MVR without TAP on the basis of propensity scores, by use of nearest-neighbor matching without replacement, and a matching tolerance (caliper) of 0.2 . The propensity score generating model had a C-statistic of 0.746 . Categorical and continuous variables between unmatched groups were compared using $\chi^{2}$ test (or Fisher exact test when the expected cell frequency was $<5$ ) and 2-sample $t$ test (or Mann-Whitney tests when the normality assumption was in doubt), respectively. Differences between the matched groups were assessed using McNemar and paired $t$ tests (or Wilcoxon signed-rank test when the normality assumption is in doubt), respectively. Survival curves were generated using the Kaplan-Meier method, and survival rate comparisons between the 2 groups were performed using the log-rank test. The stratified Cox proportional hazards model analysis was employed to estimate the treatment effect of TAP versus no TAP on late clinical outcomes of cardiac-related mortality and tricuspid valve-related events. We also considered an alternate adjustment for selection bias using the multiple Cox regression, weighted by the inverse propensity scores (IPTW). In addition to adjusting selection bias, the IPTW method enables identifying which clinical and echocardiographic parameters were significantly related to clinical outcomes. Risk factors considered in the analysis included age, sex, diabetes, etiology, hypertension, body surface area (BSA), stroke, AF, serum creatinine level, anemia, New York Heart Association functional class, surgical data, and echocardiographic parameters. Variables with $P$ values $<.2$ on univariate analysis were entered into the stepwise selection process for the multiple Cox regression model. The assumption of proportional hazards was assessed to be valid with no time dependency. All statistical analysis was done using SPSS version 21.0 (IBM-SPSS Inc, Armonk, NY).

\section{RESULTS}

\section{Baseline Characteristics and Echocardiographic Data}

The cause of valve pathology was rheumatic in 271 patients $(92.5 \%)(144$ [95.4\%] in TAP and 127 [89.4\%] in no TAP; $P=.054)$. The prevalence of preoperative $\mathrm{AF}$ was in $214(73.0 \%)(132$ [87.4\%] in TAP and 82 [57.7\%] in no TAP; $P<.001)$. Before matching, we observed that the prevalence of preoperative AF, serum creatinine level, and BSA were different between the groups. After propensity score matching, no differences in demographic data were observed between groups (Table 1). In echocardiographic data, TR grade, LAD, and right ventricular systolic pressure were also significantly greater in the TAP group. These are natural characteristics of the TAP group and will not be used in matching the data. Therefore, even after matching, TR grade and right ventricular systolic pressure remained significantly higher in the TAP group $(P<.001$ and $P=.021$, respectively), and there was a trend for increased LAD in the TAP group $(P=.080)$ (Table 2). Tricuspid annular diameter (TAD) data were available in only 60 patients (33\%) after matching. There was no difference in TAD between groups (3.27 cm [Q1-Q3, 2.97-3.74 $\mathrm{cm}]$ in the TAP group and $3.09 \mathrm{~cm}$ [Q1-Q3, 2.88-3.43 $\mathrm{cm}]$ in the no TAP group; $P=.191)$. TAD/BSA did not differ between groups $\left(2.06 \mathrm{~cm} / \mathrm{m}^{2}\right.$ [Q1-Q3, $1.77-2.22 \mathrm{~cm} / \mathrm{m}^{2}$ ] in the TAP group and $1.96 \mathrm{~cm} / \mathrm{m}^{2}$ [Q1-Q3, $1.76-2.09 \mathrm{~cm} / \mathrm{m}^{2}$ ] in the no TAP group; $P=.234$ ). 
TABLE 1. Baseline characteristics of patients with and without tricuspid annuloplasty (TAP)

\begin{tabular}{|c|c|c|c|c|c|c|}
\hline \multirow[b]{2}{*}{ Characteristic } & \multicolumn{3}{|c|}{ Total population } & \multicolumn{3}{|c|}{ Propensity-matched population } \\
\hline & $\begin{array}{c}\text { no TAP } \\
(n=142)\end{array}$ & $\begin{array}{c}\text { TAP } \\
(n=151)\end{array}$ & $P$ value & $\begin{array}{l}\text { no TAP } \\
(n=91)\end{array}$ & $\begin{array}{c}\text { TAP } \\
(n=91)\end{array}$ & $P$ value \\
\hline Age (y) & $50(41-59)$ & $52(44-59)$ & .203 & $50(41-60)$ & $49(42-58)$ & .656 \\
\hline Female sex & $92(64.8)$ & $96(63.6)$ & .903 & $55(60.4)$ & $51(56.0)$ & .644 \\
\hline $\begin{array}{l}\text { New York Heart Association functional } \\
\text { class III or IV }\end{array}$ & $41(28.9)$ & $32(21.2)$ & .129 & $24(26.4)$ & $23(25.3)$ & $>.99$ \\
\hline Hypertension & $17(12.0)$ & $16(10.6)$ & .710 & $9(9.9)$ & $8(8.8)$ & $>.99$ \\
\hline Diabetes mellitus & $15(10.6)$ & $12(7.9)$ & .545 & $11(12.1)$ & $9(9.9)$ & .824 \\
\hline History of stroke & $27(19.0)$ & $21(13.9)$ & .238 & $16(17.6)$ & $16(17.6)$ & $>.99$ \\
\hline Rheumatic etiology & $127(89.4)$ & $144(95.4)$ & .054 & $83(91.2)$ & $87(85.6)$ & .388 \\
\hline Anemia* & $30(21.1)$ & $28(18.5)$ & .579 & $15(16.5)$ & $14(15.4)$ & $>.99$ \\
\hline Serum creatinine level $(\mathrm{mg} / \mathrm{dl})$ & $0.9(0.8-1.1)$ & $0.9(0.8-1.0)$ & .013 & $1.0(0.8-1.1)$ & $1.0(0.8-1.1)$ & .531 \\
\hline Atrial fibrillation & $82(57.7)$ & $132(87.4)$ & $<.001$ & $71(78.0)$ & $72(79.1)$ & $>.99$ \\
\hline $\begin{array}{l}\text { European System for Cardiac Operative Risk } \\
\text { Evaluation score }\end{array}$ & $3.0(3.0-5.0)$ & $3.0(3.0-5.0)$ & .978 & $3.0(3.0-5.0)$ & $3.0(3.0-5.0)$ & .963 \\
\hline Body surface area $\left(\mathrm{m}^{2}\right)$ & $1.58(1.48-1.72)$ & $1.63(1.51-1.74)$ & .025 & $1.59(1.49-1.73)$ & $1.66(1.51-1.75)$ & .054 \\
\hline Predominant mitral regurgitation & $52(36.6)$ & $45(29.8)$ & .215 & $24(24.4)$ & $32(35.2)$ & .163 \\
\hline
\end{tabular}

Values are presented as median (Q1-Q3) or $\mathrm{n}(\%)$. TAP, Tricuspid annuloplasty. *Defined as hemoglobin $<12(\mathrm{~g} / \mathrm{dl})$.

\section{Operative Data}

Bileaflet mechanical valves were implanted in all patients: On- $X$ valves (On-X Life Technologies Inc, Austin, TX) in $62(27.9 \%)$, ATS valves (ATS Medical, Minneapolis, Minn) in 64 (28.8\%), St Jude Medical valves (St. Jude Medical, St. Paul, Minn) in 54 (24.3\%), and Sorin standard valves (Sorin Group, Milan, Italy) in $2(1 \%)$. For the ring annuloplasty, St Jude Medical Tailor rings (St. Jude Medical) were implanted in 50 patients $(83.4 \%)$, MC3 rings (Edwards Lifesciences, Irvine, Calif) in $6(10 \%)$, Carpentier Edwards rings (Edwards Lifesciences) in 2 $(3.3 \%)$, and Duran rings (Medtronic Inc, Minneapolis, $\mathrm{MN})$ in $2(3.3 \%)$. The cardiopulmonary bypass time (116 minutes [Q1-Q3, 100-133 minutes] in TAP and 104 minutes [Q1-Q3, 81-123 minutes] in no TAP; $P=.001$ ) and the aortic crossclamp time (96 minutes [Q1-Q3, 81-113 minutes] in TAP and 78 minutes [Q1-Q3, 56-96 minutes] in no TAP; $P<.001)$ were significantly longer in the TAP group. Concomitant maze procedure was performed in 87 patients $(47.8 \%)$. Although the maze procedure was more frequently performed in the TAP group $(65.9 \%$ in TAP and $29.7 \%$ in no TAP; $P<.001)$, the prevalence of $\mathrm{AF}$ at discharge did not differ between groups $(26.4 \%$ in TAP and $36.3 \%$ in no TAP; $P=.211)$.

\section{Early Outcomes}

There was no early mortality in either group. There were 2 bleeding episodes needing reoperation and 1 pacemaker insertion in the no TAP group. However, there were no bleeding complications and heart blocks requiring pacemaker insertion in the TAP group. The length of intensive care unit stay was not significantly different between 2 groups (1 day [Q1-Q3, 1-2 days] in TAP and 2 days [Q1-Q3, 1-3 days] in no TAP; $P=.613$ ), but the hospital stay was significantly longer in no TAP group (9 days [Q1-Q3, 7-12 days] in TAP and 11 days [Q1-Q3, $8-17$ days] in no TAP; $P<.001$ ).

TABLE 2. Preoperative echocardiographic data of patients with and without tricuspid annuloplasty (TAP)

\begin{tabular}{|c|c|c|c|c|c|c|}
\hline \multirow[b]{2}{*}{ Echocardiographic data } & \multicolumn{3}{|c|}{ Total population } & \multicolumn{3}{|c|}{ Propensity-matched population } \\
\hline & $\begin{array}{c}\text { no TAP } \\
(n=142) \\
\end{array}$ & $\begin{array}{c}\text { TAP } \\
(\mathbf{n}=\mathbf{1 5 1}) \\
\end{array}$ & $P$ value & $\begin{array}{l}\text { no TAP } \\
(n=91) \\
\end{array}$ & $\begin{array}{c}\text { TAP } \\
(\mathbf{n}=91) \\
\end{array}$ & $P$ value \\
\hline Tricuspid regurgitation grade & $0.6 \pm 0.5$ & $0.8 \pm 0.4$ & $<.001$ & $0.6 \pm 0.5$ & $0.8 \pm 0.4$ & $<.001$ \\
\hline Left ventricular ejection fraction $(\%)$ & $56(51-61)$ & $56(51-61)$ & .540 & $56(51-61)$ & $56(51-61)$ & .772 \\
\hline Left atrial diameter $(\mathrm{mm})$ & $54(50-60)$ & $60(55-68)$ & $<.001$ & $56(51-64)$ & $59(54-65)$ & .080 \\
\hline Left atrial volume index $\left(\mathrm{mL} / \mathrm{m}^{2}\right)$ & $79(60-102)$ & $94(73-122)$ & .069 & $81(66-101)$ & $83(68-111)$ & .284 \\
\hline Left ventricular end diastolic dimension $(\mathrm{mm})$ & $53(48-59)$ & $53(49-59)$ & .833 & $51(46-59)$ & $53(48-60)$ & .723 \\
\hline Left ventricular end systolic dimension (mm) & $35(31-39)$ & $36(31-39)$ & .895 & $35(30-39)$ & $36(31-40)$ & .982 \\
\hline Right ventricular systolic pressure $(\mathrm{mm} \mathrm{Hg})$ & $40(32-47)$ & $41(36-51)$ & .051 & $38(32-47)$ & $42(37-56)$ & .021 \\
\hline
\end{tabular}

Values are presented as mean \pm standard deviation or median (Q1-Q3). TAP, Tricuspid annuloplasty. 

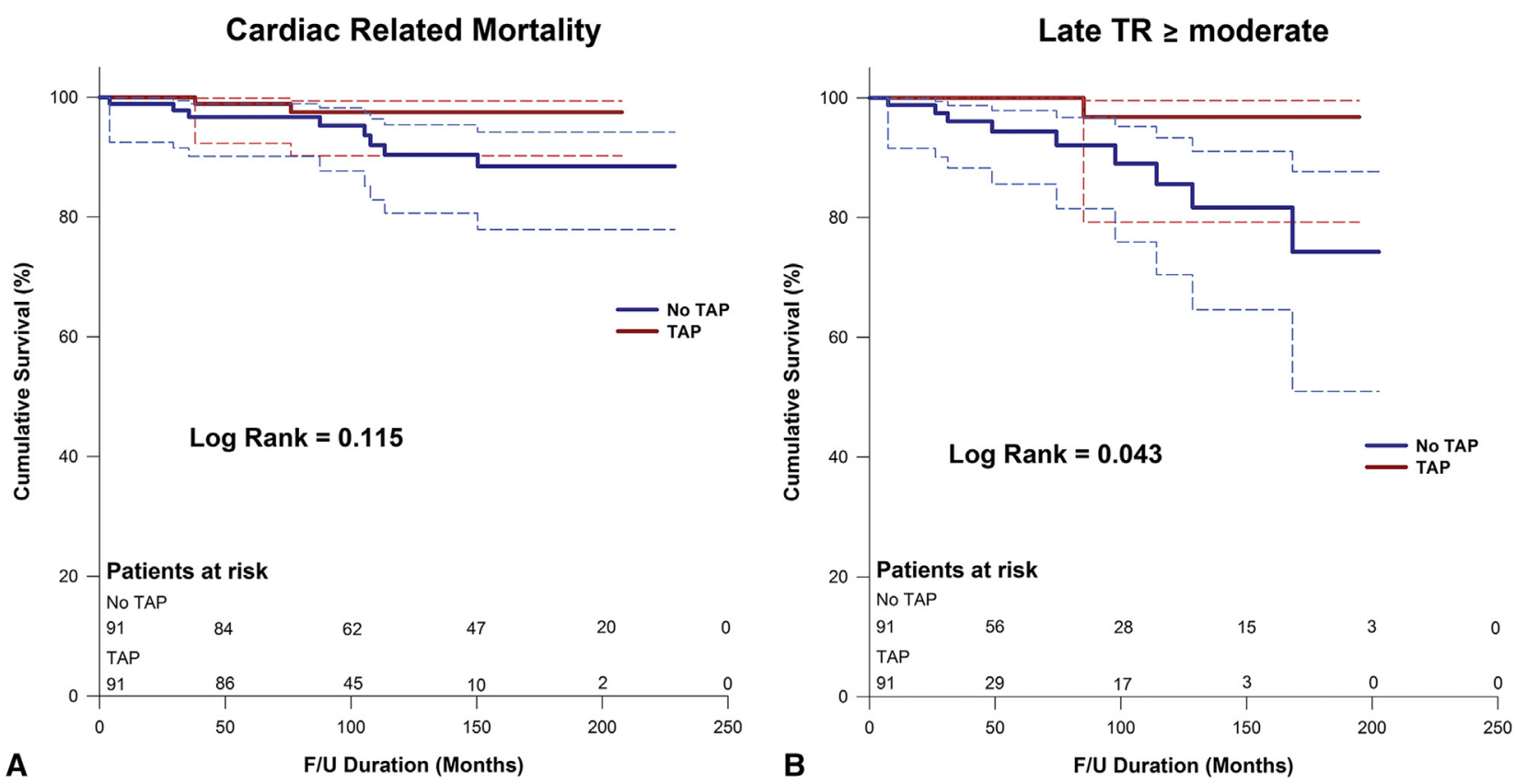

FIGURE 2. Kaplan-Meier curves of the matched tricuspid annuloplasty (TAP) and no TAP group with appropriately colored $95 \%$ confidence curves. A, Cardiac-related mortality. B, Late moderate-or-greater TR. $T R$, Tricuspid regurgitation; $F / U$, follow-up.

\section{Late Clinical Outcomes}

There were a total of 15 late deaths in both groups combined in the matched cohorts. Causes of death included 1 heart failure, 3 cerebral hemorrhages, 1 respiratory failure, 3 sepsis, 2 malignancies, and 5 unknown. Freedom from overall 10 -year mortality (5 deaths, $91.7 \%$ in TAP and 10 deaths $89.4 \%$ in no TAP at year $10 ; P=.519)$ and freedom from cardiac-related 10 -year mortality ( 2 deaths, $97.5 \%$ in TAP and 8 deaths, $90.4 \%$ in no TAP at year 10 ; $P=.115$ ) (Figure 2, A) did not differ between groups. The IPTW adjusted multiple Cox regression indicated that age (hazard ratio [HR], 1.07; 95\% confidence interval [CI], 1.02-1.12; $P=.01$ ), diabetes mellitus (HR, 6.55; 95\% CI, 2.81-15.26; $P<.001$ ), being mitral regurgitation dominant (HR, 5.55; 95\% CI, 2.45-12.56; $P<.001$ ), AF

TABLE 3. Predictors of cardiac-related mortality in a multiple Cox regression adjusted by weight of inverse probability of treatment weighted

\begin{tabular}{lccr}
\hline \multicolumn{1}{c}{ Variables } & $\begin{array}{c}\text { Hazard } \\
\text { ratio }\end{array}$ & $\begin{array}{c}\mathbf{9 5} \% \text { Confidence } \\
\text { interval }\end{array}$ & $\boldsymbol{P}$ value \\
\hline Age & 1.065 & $1.015-1.117$ & .010 \\
Diabetes mellitus & 6.547 & $2.808-15.263$ & $<.001$ \\
Mitral regurgitation dominant & 5.546 & $2.448-12.564$ & $<.001$ \\
Atrial fibrillation at discharge & 3.033 & $1.322-6.959$ & .009 \\
History of stroke & 3.910 & $1.520-10.057$ & .005 \\
No tricuspid annuloplasty & 1.225 & $0.525-2.857$ & .639 \\
\hline
\end{tabular}

From the matched data, we obtained the hazard ratio between no tricuspid annuloplasty versus tricuspid annuloplasty of 3.300 (95\% confidence interval, 0.688 $15.829 ; P=.136$ ) for cardiac-related mortality. But there were only 2 deaths in the tricuspid annuloplasty group, as opposed to 8 in the no tricuspid annuloplasty group. at discharge (HR, 3.03; 95\% CI, 1.32-6.96; $P=.009)$, and history of stroke (HR, 3.91; 95\% CI, 1.52-10.06; $P=.005)$ were risk factors independently predicting cardiac-related mortality (see Table 3 ). It also confirmed that TAP did not appear to improve cardiac-related mortality (HR, $1.23 ; P>.2$ ).

Although marginally significant, freedom from tricuspid valve-related events did not differ between groups $(84.8 \%$ in TAP and $77.5 \%$ in no TAP at year $10 ; P=.087$ ). Matched analysis also showed that there was no significance of TAP in tricuspid valve-related events (HR, $0.53 ; P=.084)$.

\section{Serial Changes of TR}

Late TR progression (moderate or greater) was observed in 10 patients ( 1 in TAP and 9 in no TAP). Freedom from recurrence of $\mathrm{TR} \geq 2$ at 10 years was significantly higher in the TAP group $(96.8 \%$ in TAP and $85.6 \%$ in no TAP at 10 years; $P=.043$ ) (Figure $2, B$ ).

Figure 3 showed the subgroup analysis with KaplanMeier survival curve for late moderate-or-greater TR of the TAP versus no TAP groups according to the postoperative rhythm status at discharge. Freedom from TR recurrence did not differ between groups in patients with AF at discharge $(P=.460)$ (Figure $3, A)$. However, freedom from TR recurrence was significantly higher in the TAP group in patients with sinus rhythm at discharge $(P=.047)$ (Figure 3, B).

Figure 4 showed the serial changes of the proportion of preoperative, postoperative (at discharge), and last followup (more than 5 years) TR grade in each group. The 
Late $T R \geq$ moderate

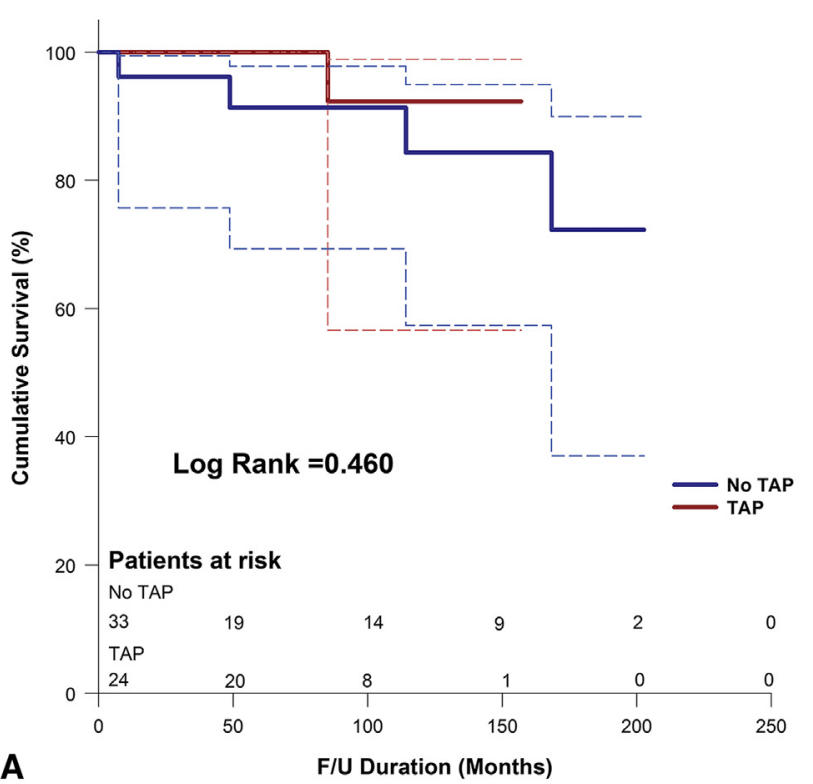

Late $T R \geq$ moderate

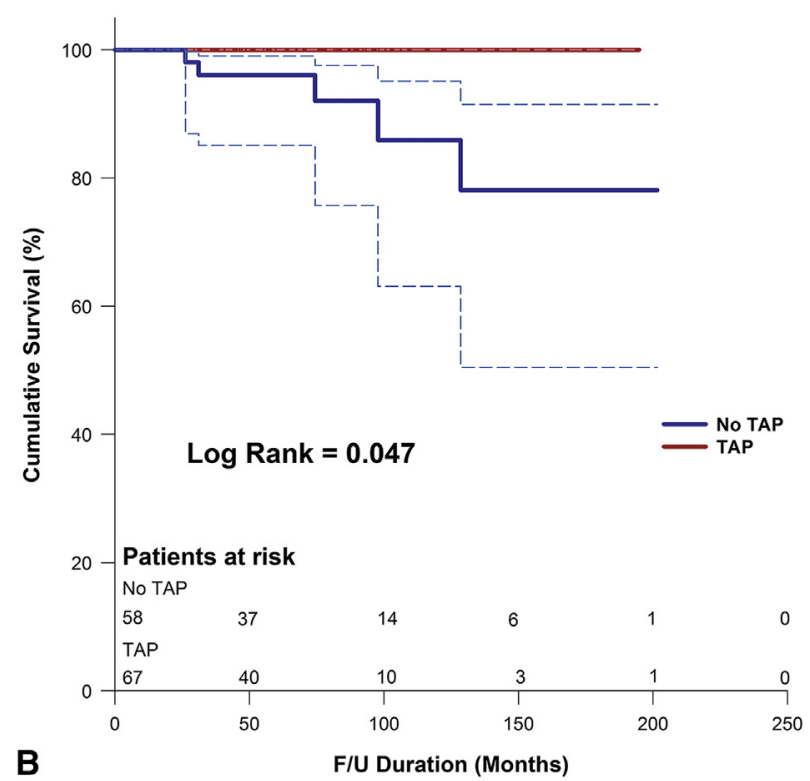

FIGURE 3. Kaplan-Meier curves for late moderate-or greater tricuspid regurgitation $(T R)$ of the matched tricuspid annuloplasty (TAP) and no TAP groups according to postoperative atrial fibrillation and sinus rhythm with appropriately colored $95 \%$ confidence curves. A, Late moderate-or-greater TR in postoperative atrial fibrillation. B, Late moderate-or-greater TR in postoperative sinus rhythm. $F / U$, follow-up.

median time to diagnosis of moderate-or-greater TR was 68 months (Q1-Q3, 35-99 months). There was 1 patient with moderate TR in the TAP group at discharge. However, there were several patients with moderate-or-greater TR in the no TAP group at discharge (the incidence of moderate-or-greater TR was $1.1 \%$ in the TAP group and $6.6 \%$ in the no TAP group; $P=.125)$. The 1 late

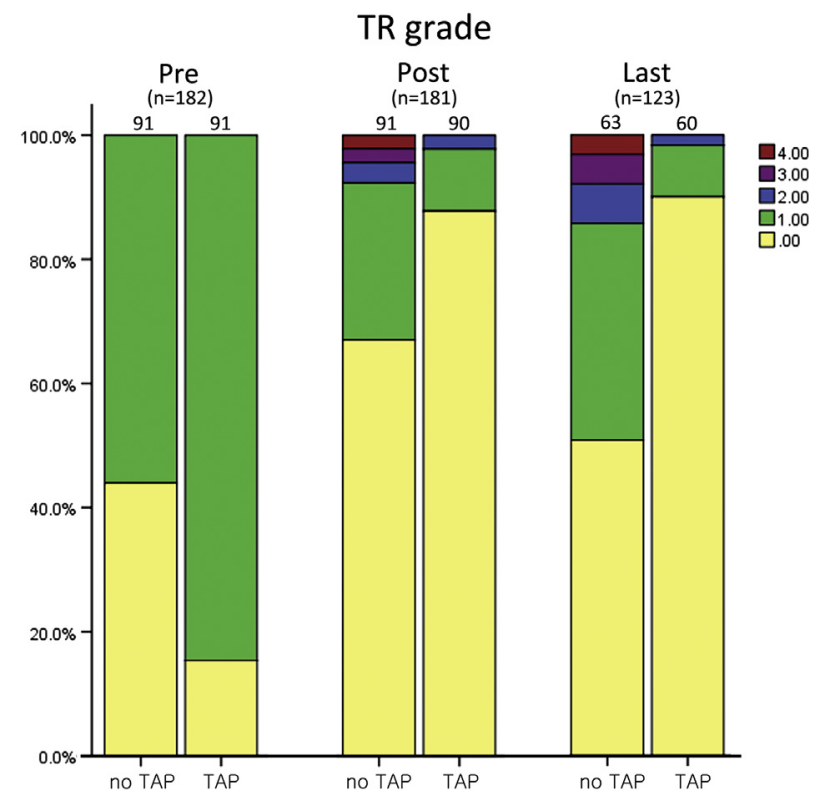

FIGURE 4. The serial changes of the proportion of preoperative, postoperative (at discharge) and last follow-up (more than 5 years) tricuspid regurgitation (TR) grade in each group. TAP, Tricuspid annuloplasty. moderate-or-greater TR in the TAP group occurred in a patient who underwent suture annuloplasty $(3.2 \% ; 1$ out of 31 patients). There was no late moderate-or-greater TR among patients who underwent ring annuloplasty (0 out of 60 patients).

Three patients in the no TAP group required concomitant tricuspid valve operation at the time of reoperation; 1 patient due to progression of TR (severe), and 2 patients due to associated AF with minimal to mild TR. However, there were no tricuspid valve reoperations in the TAP group.

\section{DISCUSSION}

Tricuspid valve repair is recommended for severe TR in patients with mitral valve disease requiring mitral valve surgery (class I) under current clinical guidelines. The 2014 American College of Cardiology/American Heart Association updated guidelines ${ }^{5}$ state that tricuspid valve repair can be beneficial for mild, moderate, or greater functional TR during mitral valve surgery for patients with tricuspid annular dilatation (class IIa) or prior evidence of right-side heart failure (class IIa), and that tricuspid valve repair may be considered for pulmonary hypertension (class IIb). According to the 2012 European Society of Cardiology/European Society for Cardio-Thoracic Surgery guidelines, ${ }^{4}$ tricuspid valve repair should be considered in patients with mild-to-moderate TR and a dilated tricuspid annulus $\left(\geq 40 \mathrm{~mm}\right.$ or $\left.>21 \mathrm{~mm} / \mathrm{m}^{2}\right)$ during mitral valve surgery (class IIa). Despite the aforementioned guidelines, surgical indication of prophylactic TAP for patients with less-than-moderate TR at the time of left-side valve surgery 
remains controversial because multiple complex factors are associated with TR, such as etiology (degenerative, rheumatic), concomitant AF, unreliable TR grading, or measuring of TAD (echocardiographic, direct). ${ }^{6,7}$ There have been reports that TR with mitral valve disease does not progress after mitral valve surgery. ${ }^{19-21}$ On the other hand, several authors have suggested that there are beneficial effects of TAP for patients with less-thanmoderate TR. ${ }^{8,10,11}$ Dreyfus and colleagues ${ }^{8}$ reported that late TR grade was significantly lower in their TAP group $(P=.001)$ in patients with tricuspid annular dilatation $(\geq 70 \mathrm{~mm})$ who underwent mitral valve repair compared with patients without tricuspid annular dilatation. Their study included some patients with moderate-or-greater TR (18 out of 148 patients; $12.2 \%$ ) and there was no description of the maze procedure. Benedetto and colleagues ${ }^{10}$ reported results of a randomized study in 44 patients (22 TAP vs 22 no TAP) undergoing mitral valve surgery. They showed that TR grade was significantly higher in their no TAP group at 12-month follow-up $(P=.007)$. Kim and colleagues ${ }^{11}$ reported that upon subgroup analysis, freedom from moderate-to-severe TR was higher in the TAP group than in the no TAP group among the patients with preoperative mild TR $(P=.038)$ in patients who underwent mechanical MVR. Despite the findings of previous reports, conclusive long-term clinical results of prophylactic TAP for patients with less-than-moderate TR are limited.

Our study demonstrated the beneficial effect of prophylactic TAP in patients with less-than-moderate TR who underwent mechanical MVR via propensity scorematched analysis. TAP can be performed safely without increases in early mortality and morbidities, including heart block. We also found that prophylactic TAP can prevent progression of late moderate-or-greater TR. TAP showed a tendency to prevent tricuspid valve-related events and was marginally significant $(P=.087)$.

Untreated TR has been suggested to be a risk factor for TR progression and worse clinical outcomes. ${ }^{8,11,22}$ Table 3 summarized the HRs between the 2 groups for cardiac-related mortality. The propensity-matched data indicated marginal evidence that there appears to be no difference between the 2 groups in TAP and cardiac-related mortality (HR, 3.30; $P=.136$ see in Table 3). These statistical results could be mainly due to chance with a small number of deaths. However, it seems plausible to anticipate a long-term trend of a beneficial TAP effect (see Figure 2, $A$ ). Although TAP had a lack of significance for tricuspid valve-related events, matched analysis demonstrated that TAP showed marginal evidence for preventing tricuspid valve-related events (HR, $0.53 ; P=.084)$. TAP also showed a trend of a long-term beneficial effect on tricuspid valve-related events $(P=.087)$.

In our retrospective observation study, preoperative TR grade and right ventricular systolic pressure were significantly higher, and there was a trend for greater LAD in the TAP group based on the propensity scorematched analysis. These findings may be a reflection of greater right atrium size and more severe right ventricular loading in the TAP group. Current guidelines posit that tricuspid annular dilatation is more important than TR grade for prophylactic surgical intervention, because the severity of TR changes under different conditions. Unfortunately, we did not have data for TAD measurements during the operations. Preoperative TAD was measured during the later period of our study and available in only 60 patients $(33 \%)$ after matching. Although TAD was slightly greater in the no TAP group, we did not find any statistical difference in TAD between groups (3.27 cm [Q1-Q3, 2.97$3.74 \mathrm{~cm}$ ] in TAP and $3.09 \mathrm{~cm}$ [Q1-Q3. 2.88-3.43 cm] in no TAP; $P=.191$ ). Previously, the indication of TAP was suggested in patients with TAD/BSA $>21 \mathrm{~mm} / \mathrm{m}^{2}{ }^{22}$ TAD/BSA in our study was slightly smaller in both groups compared with the previous suggestion $\left(2.06 \mathrm{~cm} / \mathrm{m}^{2}\right.$ [Q1Q3, $1.77-2.22 \mathrm{~cm} / \mathrm{m}^{2}$ ] in TAP and $1.96 \mathrm{~cm} / \mathrm{m}^{2}$ [Q1-Q3, $\left.1.76-2.09 \mathrm{~cm} / \mathrm{m}^{2}\right]$ in no TAP; $\left.P=.234\right)$. Nevertheless, progression of TR was observed in the no TAP group. TAD $>40 \mathrm{~mm}$ or TAD/BSA $>21 \mathrm{~mm} / \mathrm{m}^{2}$ have been suggested as indications for TAP by several authors. ${ }^{10,22,23}$ This may not be routinely applied in all patients with TR. The results of our study show that it should be lower for patients with rheumatic etiology, as David and colleagues $^{7}$ suggested in recent report. In addition, there was only 1 late TR recurrence (3.2\%) in the suture annuloplasty group (0 in the ring annuloplasty group). We are inclined to suggest that the modified De Vega technique with multiple pledgets may have a preventive role for TR progression in prophylactic TAP for less-than-moderate TR.

$\mathrm{AF}$ is a well-known risk factor for late TR progression and worsens late outcomes. Several authors suggest that maze operation can have a preventive effect on late TR progression. $^{24-26}$ In our study, AF at discharge was an independent risk factor for cardiac-related mortality (Table 3). In subgroup analysis, the effects of TAP on recurrence of moderate-or-greater TR were prominent in patients with sinus rhythm compared with patients with $\mathrm{AF}$ at discharge (Figure 3). These findings may reflect that the maze procedure is more important than prophylactic TAP in improving late outcomes in mitral valve disease with AF. Further prospective randomized study is necessary to evaluate the benefit of additional TAP with maze procedure during mitral valve surgery.

There were several limitations of our study. First, our study was retrospective in nature. Surgical indication and technique of MVR, TAP, and maze procedure changed over the long duration of the study. The maze procedure and prophylactic TAP were not popular in the early period of our study. Several surgeons were involved with performing the TAP during the long period of our study. 
The prevalence of $\mathrm{AF}$ and the maze operation were significantly different between groups. Therefore, propensity score matching was applied to minimize the selection bias. Based on the propensity score-matched group analysis, we find that there was no difference in the prevalence of preoperative $\mathrm{AF}$ and the incidence of $\mathrm{AF}$ at discharge. Second, accurate measurement of TAD, right atrial dimension, and right-side heart function were not routinely evaluated. Therefore, most TAP operations were determined based on the clinical decisions of each surgeon. Finally, follow-up echocardiographic data were not available for all patients; thus it is possible that sampling bias may have affected long-term hemodynamic parameters.

\section{CONCLUSIONS}

Prophylactic TAP for patients with less-than-moderate TR who underwent mechanical MVR for mitral valve disease (rheumatic 92.5\%, AF 73\%) prevent late recurrence of significant TR without increasing early morbidities or mortality. Although prophylactic TAP showed marginal clinical benefits for patients with less-than-moderate TR, we believe it is plausible to anticipate a long-term trend of a benefit of TAP in longerterm follow-up studies. Many other longer-term prospective randomized studies are needed to confirm our study findings and to ascertain clinical benefits of prophylactic TAP during mitral valve surgery.

\section{Conflict of Interest Statement}

Authors have nothing to disclose with regard to commercial support.

\section{References}

1. King RM, Schaff HV, Danielson GK, Gersh BJ, Orszulak TA, Piehler JM, et al. Surgery for tricuspid regurgitation late after mitral valve replacement. Circulation. 1984;70:I193-7.

2. Kwon DA, Park JS, Chang HJ, Kim YJ, Sohn DW, Kim KB, et al. Prediction of outcome in patients undergoing surgery for severe tricuspid regurgitation following mitral valve surgery and role of tricuspid annular systolic velocity. Am J Cardiol. 2006;98:659-61.

3. Nath J, Foster E, Heidenreich PA. Impact of tricuspid regurgitation on long-term survival. J Am Coll Cardiol. 2004;43:405-9.

4. Vahanian A, Alfieri O, Andreotti F, Antunes MJ, Baron-Esquivias G, Baumgartner $\mathrm{H}$, et al. Guidelines on the management of valvular heart disease (version 2012): the Joint Task Force on the Management of Valvular Heart Disease of the European Society of Cardiology (ESC) and the European Association for Cardio-Thoracic Surgery (EACTS). Eur J Cardiothorac Surg. 2012;42: S1-44.

5. Nishimura RA, Otto CM, Bonow RO, Carabello BA, Erwin JP III, Guyton RA, et al. 2014 AHA/ACC Guideline for the Management of Patients With Valvular Heart Disease: a report of the American College of Cardiology/American Heart Association Task Force on Practice Guidelines. Circulation. 2014;129:e521-643.

6. Dion RA. Is the air in Toronto, Rochester, and Cleveland different from that in London, Monaco, Leiden, Genk, Milan, and New York? J Thorac Cardiovasc Surg. 2015;150:1040-3.

7. David TE, David CM, Manhiolt C. When is tricuspid valve annuloplasty necessary during mitral valve surgery? J Thorac Cardiovasc Surg. 2015;150:1043-4.
8. Dreyfus GD, Corbi PJ, Chan KM, Bahrami T. Secondary tricuspid regurgitation or dilatation: which should be the criteria for surgical repair? Ann Thorac Surg. 2005;79:127-32.

9. Calafiore AM, Gallina S, Iaco AL, Contini M, Bivona A, Gagliardi M, et al. Mitral valve surgery for functional mitral regurgitation: should moderate-ormore tricuspid regurgitation be treated? a propensity score analysis. Ann Thorac Surg. 2009;87:698-703.

10. Benedetto U, Melina G, Angeloni E, Refice S, Roscitano A, Comito C, et al Prophylactic tricuspid annuloplasty in patients with dilated tricuspid annulus undergoing mitral valve surgery. J Thorac Cardiovasc Surg. 2012;143:632-8.

11. Kim JB, Yoo DG, Kim GS, Song H, Jung SH, Choo SJ, et al. Mild-to-moderate functional tricuspid regurgitation in patients undergoing valve replacement for rheumatic mitral disease: the influence of tricuspid valve repair on clinical and echocardiographic outcomes. Heart. 2012;98:24-30.

12. Kim H, Park PW, Sung K, Lee YT, Jun TG, Kim WS, et al. Mid-term results of the Cox maze III procedure combined with open mitral commissurotomy for the treatment of rheumatic mitral stenosis. Circ J. 2010;74:1332-8.

13. Kim YH, Lee SC, Her AY, Kim HJ, Choi JO, Shin DH, et al. Preoperative left atrial volume index is a predictor of successful sinus rhythm restoration and maintenance after the maze operation. J Thorac Cardiovasc Surg. 2007;134: 448-53.

14. Park SJ, On YK, Kim JS, Jeong DS, Kim WS, Sung K, et al. Effect of left atrial activity after the maze procedure on clinical and echocardiographic outcomes. Circ J. 2014;78:1584-92.

15. Akins CW, Miller DC, Turina MI, Kouchoukos NT, Blackstone EH Grunkemeier GL, et al. Guidelines for reporting mortality and morbidity after cardiac valve interventions. J Thorac Cardiovasc Surg. 2008;135:732-8.

16. Lang RM, Bierig M, Devereux RB, Flachskampf FA, Foster E, Pellikka PA, et al Recommendations for chamber quantification: a report from the American Society of Echocardiography's Guidelines and Standards Committee and the Chamber Quantification Writing Group, developed in conjunction with the European Association of Echocardiography, a branch of the European Society of Cardiology. J Am Soc Echocardiogr. 2005;18:1440-63.

17. Foale R, Nihoyannopoulos P, McKenna W, Kleinebenne A, Nadazdin A, Rowland E, et al. Echocardiographic measurement of the normal adult right ventricle. Br Heart J. 1986;56:33-44.

18. Zoghbi WA, Enriquez-Sarano M, Foster E, Grayburn PA, Kraft CD, Levine RA et al. Recommendations for evaluation of the severity of native valvular regurgitation with two-dimensional and Doppler echocardiography. J Am Soc Echocar diogr. 2003;16:777-802.

19. Yilmaz O, Suri RM, Dearani JA, Sundt TM III, Daly RC, Burkhart HM, et al Functional tricuspid regurgitation at the time of mitral valve repair for degenerative leaflet prolapse: the case for a selective approach. J Thorac Cardiovasc Surg. 2011;142:608-13.

20. Chan V, Burwash IG, Lam BK, Auyeung T, Tran A, Mesana TG, et al. Clinical and echocardiographic impact of functional tricuspid regurgitation repair at the time of mitral valve replacement. Ann Thorac Surg. 2009;88:1209-15.

21. Rajbanshi BG, Suri RM, Nkomo VT, Dearani JA, Daly RC, Burkhart HM, et al. Influence of mitral valve repair versus replacement on the development of late functional tricuspid regurgitation. J Thorac Cardiovasc Surg. 2014;148:1957-62.

22. Shi KH, Xuan HY, Zhang F, Xu SS, Wu JX, Cao W, et al. Evolution of tricuspid regurgitation after mitral valve surgery for patients with moderate-or-less functional tricuspid regurgitation. Heart Surg Forum. 2012;15:E121-6.

23. Goldstone AB, Howard JL, Cohen JE, MacArthur JW Jr, Atluri P, Kirkpatrick JN et al. Natural history of coexistent tricuspid regurgitation in patients with degenerative mitral valve disease: implications for future guidelines. $J$ Thorac Cardiovasc Surg. 2014;148:2802-9.

24. Matsuyama K, Matsumoto M, Sugita T, Nishizawa J, Tokuda Y, Matsuo T Predictors of residual tricuspid regurgitation after mitral valve surgery. Ann Thorac Surg. 2003;75:1826-8.

25. Stulak JM, Schaff HV, Dearani JA, Orszulak TA, Daly RC, Sundt TM III. Restoration of sinus rhythm by the Maze procedure halts progression of tricuspid regurgitation after mitral surgery. Ann Thorac Surg. 2008;86:40-4; discussion 4-5.

26. Je HG, Song H, Jung SH, Choo SJ, Song JM, Kang DH, et al. Impact of the Maze operation on the progression of mild functional tricuspid regurgitation. $J$ Thorac Cardiovasc Surg. 2008;136:1187-92.

Key Words: mitral valve, tricuspid regurgitation, valvular annuloplasty 